\title{
Investigating the Impact of COVID-19 Pandemic on Mental Health Status and Factors Influencing Negative Mental Health among Health-Care Workers in Dubai, United Arab Emirates
}

\author{
Fatma AlGhufli ${ }^{a}$ Rayyan AIMulla ${ }^{a}$ Ola Alyedi ${ }^{a}$ Sham Zain AlAbdin ${ }^{b}$ \\ Mohammed Moutaz Nakhalc \\ aFamily Medicine Department, PHCS, AI Khawaneej Health Centre, Dubai Health Authority, Dubai, UAE; \\ bPharmacology Department, College of Medicine and Health Sciences, United Arab Emirates University, Al-Ain, UAE; \\ 'Biochemistry Department, College of Medicine and Health Sciences, United Arab Emirates University, Al-Ain, UAE
}

\section{Keywords}

Health-care workers · COVID-19 · Coronavirus · Depression · Anxiety · Stress · Insomnia $\cdot$ Psychological health

\begin{abstract}
Aim: This study aimed to investigate the prevalence of psychological health outcomes and associated factors among health-care workers (HCWs) during COVID-19 pandemic. Subject and Methods: This observational cross-sectional study was conducted on 550 HCWs at 4 different clinics (fever, clean, telemedicine, and mixed clinics) in Dubai Health Authority from July 2020 to September 2020. Severity of depression, anxiety, and stress was examined by calculating the Depression Anxiety Stress Scale (DASS-21) for each subscale, while insomnia was determined by Insomnia Severity Index (ISI) score. Factors associated with psychological symptoms were identified by univariate (ANOVA and independent $t$ test), and independent factors were detected by multivariate linear regression analysis after controlling other variables. $p$ value of $<0.05$ was considered with significant results. $\boldsymbol{R} \boldsymbol{e}$ sults: A total of 400 participants completed the survey. Majority of them were females $(84.5 \%, n=338)$ and aged between 18 and 31 (53.5\%, $n=214)$. About 76.5\% $(n=306)$ reported symptoms of depression, 315 (78.8\%) anxiety, 254
\end{abstract}

karger@karger.com www.karger.com/dmj

Karger $\frac{1}{\%}$
(C) 2021 The Author(s)

Published by S. Karger AG, Basel

This is an Open Access article licensed under the Creative Commons Attribution-NonCommercial-4.0 International License (CC BY-NC) (http://www.karger.com/Services/OpenAccessLicense), applicable to the online version of the article only. Usage and distribution for commercial purposes requires written permission.
(63.5\%) stress-based, and 187 (46.8\%) suffered from clinical insomnia. Frontline HCWs at fever clinic were at higher risk of developing burnout symptoms $(p<0.001)$. Furthermore, being younger, nonmarried, and nurse had no kids and changed accommodation during the crisis; all together were at higher risk of developing burnout symptoms $(p<0.05)$. Adjusted linear regression showed that age was the major independent factor affecting DASS-21 subscales without affecting the level of insomnia $(<0.01)$. In addition, being worried about the family and loved ones independently influenced higher levels of depression and anxiety-based $(<0.001)$. Conclusion: This study revealed that COVID-19 outbreak has a significant psychological impact on frontline HCWs and the most vulnerable groups to psychological distress. Efficient support and interventions are needed to protect HCWs from being infected and promote their well-being. @ 2021 The Author(s)

Published by S. Karger AG, Basel

\section{Introduction}

In December 2019, a severe acute respiratory infection "COVID-19" caused by a novel coronavirus has emerged from Wuhan, China, and spread dramatically worldwide [1]. The World Health Organization declared in March 
2020 that COVID-19 is a global pandemic and considered as a public health emergency of an international concern [2]. The widespread and rapid transmission of the virus along with the associated mortalities have contributed to enormous levels of panic and distress among the public. Locally, recent reports of the UAE documented around 38,808 COVID- 19 cases with approximately 281 deaths [3].

Beside the public panic during the COVID-19 crisis, health-care workers (HCWs) were exposed to greater risk of burnout symptoms as they have been in the frontline encountering this pandemic. Their profession put them under extreme pressure in treating infected patients, increased possibility of being infected, and fear of transmitting the infection to their families. In addition, the increased number of cases and mortality rates and depletion of personnel protection in the health-care institutions, all together were major contributing factors to affect HCWs' psychological health [4].

After examining HCWs' psychological health during previous outbreaks (as in SARS pandemic), it was found that majority of them were emotionally traumatized [5]. Several studies suggested a high rate of burnout and psychological manifestation among HCWs during the current COVID-19 crisis. A recent meta-analysis, including 13 studies reported that $23.2 \%$ of HCWs suffered from anxiety, while $22.8 \%$ of them had depression. Moreover, insomnia was prevalent among $38.9 \%$ of the HCWs.

Therefore, comprehending the influence of COVID-19 crisis on the psychological health of frontline HCWs is crucial to manage their psychological symptoms and implementing effective strategies in supporting their mental health. So, this study aimed to assess the psychological health of the frontline HCWs in Dubai and identify the most vulnerable groups among HCWs that were more likely affected by the COVID-19 pandemic.

\section{Materials and Methods}

\section{Study Design and Setting}

This observational cross-sectional study was conducted on HCWs at primary health-care centers controlled by Dubai Health Authority to assess the impact of COVID-19 pandemic on mental health during a period from 28 July to 14 September 2020. Two health centers were utilized to accommodate the screening process of COVID-19 cases and triage them through the health-care system. Those centers were called as "fever or mixed clinics," which were dedicated to assess, test, treat, and triage suspected cases during this outbreak; hence, diverting cases from the general practice and emergency departments. Another health-care center was adjusted to be a clean clinic with strict safety measures to cover Antenatal Care and Child Health Services. In addition, a controlled telemedicine facility, where physicians have no direct contact with patients and consultation is only permitted to a live video streaming. The study was approved by the Dubai Scientific Research Ethics Committee in Dubai Health Authority (DSREC-06/2020_57).

\section{Study Participants}

According to the published statistics in 2020, the total number of HCWs in the Dubai Health Authority 39,411 active HCWs, including physicians, nurses, and allied health professionals [6]. Based on the Raosoft calculator for sample size estimation, the minimum required sample for this study was 377 with a confidence interval of 95.0 and 5.0\% margin of error [7]. Assuming the response rate was $\sim 70.0-80.0 \%$, the study comprised $550 \mathrm{HCWs}$ from various specialties in the abovementioned health-care centers, including physicians, nurses, lab technicians, pharmacists, and administrative staff. An informed consent form was provided for all participants before completing their participation in this study.

\section{Study Outcomes}

The questionnaire was self-administered and diffused to a convenient sample of HCW s in the study facilities. Demographic characteristics, including gender, age, level of education, years of experience, and other social information were collected in the first part of the questionnaire. In the second and third parts, the impact of COVID-19 pandemic was assessed using 2 validated tools in the English language: Depression Anxiety Stress Scale (DASS-21) for depression, anxiety, and stress, and Insomnia Severity Index (ISI) for recognizing insomnia among the participants. The average time to complete the questionnaire including both scales was not more than $5 \mathrm{~min}$.

The study also investigated the mental health of frontline HCWs worked in the fever clinics compared to other health-care facilities (clean clinic and telemedicine physicians), whom were not on direct contact with COVID-19 patients. Moreover, it identified the most vulnerable groups to psychological distress in the health-care system.

\section{Study Tools for Assessment of Mental Health}

The DASS- 21 is a self-reporting quantitative scale with 21 items for assessment of the severity of 3 emotional dimensions: depression, anxiety, and stress. It is the short version of DASS- 42 that was developed by Lovibond and Lovibond [8]. It is based on emotional rather than categorical conception; however, cutoff points are recommended for conventional evaluation of severity. Each subscale consists of 7 items (highest loading items of DASS-42), and each item is scored on 4-point scale ranging from 0 "Not applied to me at all" to 3 "Applied to me very much." Responses of each subscale (depression, anxiety, and stress) are summed, and their total scores are recorded separately to represent the main scale (DASS-21) [8]. The severity of each emotional subscale can be determined based on different scoring systems according to Coker et al. [9]. Normal scores of depression domain range from 0 to 9 , while scores of 10-28 indicate pathological depression. For anxiety domain, score ranges of $0-7$ are considered at normal level, while higher scores from 8 to 20 reflect more severe anxiety levels. For stress domain, normal scores are within range of $0-14$, while pathological stress is detected between score range of 15-34 [9]. The scale has high reliability and advantages over the full DASS. It is shorter and free from problematic questions and more its latent structure is clearer and more focused. 
Table 1. Sociodemographic characteristics of the participants

\begin{tabular}{|c|c|}
\hline Variables & $N(\%)$ \\
\hline \multicolumn{2}{|l|}{ Gender } \\
\hline Male & $62(15.5)$ \\
\hline Female & $338(84.5)$ \\
\hline \multicolumn{2}{|l|}{ Marital status } \\
\hline Married & $336(84.0)$ \\
\hline Not married & $64(16.0)$ \\
\hline \multicolumn{2}{|l|}{ Age } \\
\hline 18-30 & $53(13.3)$ \\
\hline $31-40$ & $214(53.5)$ \\
\hline $41-50$ & $87(21.8)$ \\
\hline $51-60$ & $45(11.3)$ \\
\hline$>60$ & $1(0.3)$ \\
\hline \multicolumn{2}{|l|}{ Work place } \\
\hline Fever & $53(13.3)$ \\
\hline Clean & $123(30.8)$ \\
\hline Telemedicine & $3(0.8)$ \\
\hline Mixed & $221(55.3)$ \\
\hline \multicolumn{2}{|l|}{ Type of work } \\
\hline Physician & $64(16.0)$ \\
\hline Nurse & $184(46.0)$ \\
\hline Pharmacist & $3(0.8)$ \\
\hline Lab technician & $136(34.0)$ \\
\hline Radiologist & $6(1.5)$ \\
\hline Administrative staff & $5(1.3)$ \\
\hline Medical record staff & $2(0.5)$ \\
\hline \multicolumn{2}{|l|}{ Do you have kids? } \\
\hline Yes & $308(77.0)$ \\
\hline No & $92(23.0)$ \\
\hline
\end{tabular}

The ISI is a 7-item screening tool of the nature, symptoms, and severity of insomnia using a Likert scale. The items of the questionnaire represent the subjective quality of sleeping patterns, distress caused by sleeping disorders, and the degree that insomnia affects daily functioning. There are 4 categories for classifying the severity of the insomnia as the following: scores of $0-7$ "No significant insomnia symptoms," 8-14 "Sub-threshold insomnia," 15-21 "moderately severe insomnia," and 22-28 "Severe insomnia" [10].

\section{Statistical Analysis}

Statistical analysis was performed using the SPSS (version 23.0). Descriptive analysis included frequencies and percentages for categorical variables, while mean \pm standard deviation for continuous measures (DASS-21 and ISI scores). Univariate analysis was carried out to identify the associations between the psychological symptoms and related factors by conducting the following tests: student's $t$ test and 1- way ANOVA. Multivariate linear regression analysis was conducted to identify independently significant factors affecting the psychological measurements after controlling all significant factors in univariate analysis. The statistical significance of $\leq 0.05$ was considered in the univariate analysis.

The Impact of COVID-19 on Health-Care Workers' Mental Health

Yes

No

Maybe, but did not do the test

$14(3.5)$

Do you think that COVID-19 pandemic affected your personality?

Yes $240(60.0)$

No $\quad 60(15.0)$

Partially $100(25.0)$

Were you worried about your loved one and family during this pandemic?

$\begin{array}{ll}\text { Yes } & 352(88.0) \\ \text { No } & 16(4.0) \\ \text { Partially } & 32(8.0)\end{array}$

Did you change your accommodation due to COVID-19 pandemic?

Yes $83(20.8)$

No $288(72.0)$

Partially $29(7.3)$

\section{Results}

\section{Sociodemographics of the Participants}

Table 1 represents the sociodemographic characteristics of the participants. A total of $400 \mathrm{HCW}$ s completed this study (response rate $\sim 72.7 \%$ ). Majority of them were females $(84.5 \%, n=338)$ and were married $(84.0 \%, n=$ 336). More than half of the HCWs aged between 31 and 40 years old $(53.5 \%, n=214)$ and were working in mixed clinics $(55.3 \%, n=221)$. Nursing was the most common profession among the HCWs $(46.0 \%, n=184)$ followed by lab technicality $(34.0 \%, n=136)$. Most of the HCWs had kids $(77.0 \%, n=308)$ and underwent the distance learning program $(60.3 \%, n=241)$. Around $57.8 \%(n=$ 231) of the HCWs worked directly with COVID-19 patients, and only $13.8 \%(n=55)$ reported that they got infected with virus. Majority of the HCWs reported that COVID-19 pandemic affected their personality $(60.0 \%$, $n=240$ ), and they were worried about their loved ones and families $(88.0 \%, n=352)$. Only less than quarter of the HCWs changed their accommodations due to COVID-19 crisis $(20.8 \%, n=83)$. 
Table 2. Mean scores \pm SD of the psychometric instruments (DASS21 and $|S|)$

\begin{tabular}{llll}
\hline Instrument & Mean & SD & Range \\
\hline DASS-21 depression & 11.9 & 8.5 & 37 \\
DASS-21 anxiety & 11.1 & 8.3 & 38 \\
DASS-21 stress & 10.0 & 8.0 & 36 \\
ISI & 7.8 & 6.0 & 28 \\
\hline
\end{tabular}

SD, standard deviation; DASS-21, Depression Anxiety Stress Scale 21; ISI, Insomnia Severity Index.

\section{Prevalence of Mental Health Problems during} COVID-19 Crisis

Mean scores \pm standard deviation of the psychometric instruments (DASS-21 and ISI) are represented in Table 2. Among all HCWs, 306 (76.5\%) of them reported symptoms of depression, $315(78.8 \%)$ anxiety, and 254 (63.5\%) stress based on DASS-21 scale. In addition, 187 (46.8\%) suffered from clinical insomnia (mild, moderate, and severe). Further details on the prevalence of depression, anxiety, stress, and insomnia at different levels of severity are shown in Table 3.

\section{Prevalence of Mental Health Problems at Different}

Types of Clinics during COVID-19 Crisis

Among the HCWs working at fever clinic, 46 (86.8\%), $40(75.5 \%), 33(62.3 \%)$, and $18(34.0 \%)$ of them had depression, anxiety, stress, and insomnia, respectively. While 87 (70.7\%), 97 (78.9\%), 79 (64.2\%), and 64 (52.0\%) had the abovementioned mental health disorders in the clean clinic in the same manner. The mixed clinic had 170 (76.9\%) of HCWs with depression, $176(79.6 \%)$ with anxiety, 140 (63.3\%) distressed, and 103 (46.6\%) with insomnia. On the other side, among the telemedicine clinic's HCWs, all of them suffered from depression (100.0\%, $n=3$ ), and only 2 of them (66.7\%) suffered from anxiety, stress, and insomnia.

Figures 1-4 show the prevalence of psychological disorders among fever, clean, and mixed clinics. Majority of HCWs in the fever clinic had extremely severe depression $(45.3 \%, n=24)$ and anxiety $(54.7 \%, n=29)$, with moderate level of stress $(22.6 \%, n=12)$ and sub-threshold insomnia $(26.4 \%, n=14)$. While at the clean and mixed clinics, HCWs had extremely severe levels of depression $(30.1 \%, n=37)$, anxiety $(54.5 \%, n=67)$, and stress $(20.3 \%$, $n=25)$ with subthreshold insomnia $(38.2 \%, n=47)$. Among the $3 \mathrm{HCWs}$ working at telemedicine clinic, 2 reported extremely severe depression, moderate to severe anxiety, and stress (66.7\%); however, insomnia levels were between subthreshold and moderately severe (33.3\%, $n=1$ for each category).

\section{Predictors of Mental Health Status during COVID-19 Crisis}

Table 4 represents univariate analysis for psychological symptoms of depression, anxiety, stress, and insomnia among the HCWs during the COVID-19 pandemic. It was found that HCWs at age range of 18-30 years old were more likely to be depressed ( $p=0.003$ ), anxious $(p=0.01)$, and stressed $(p=0.003)$ than other age-groups. Nurses and nonmarried HCWs reported more symptoms of depression ( $p=0.03$ and $p<0.001$, respectively). In addition, HCWs working at fever clinics showed higher levels of depression $(p<0.001)$ and stress $(p=0.03)$ than HCWs working at other clinics. Also, those who were directly working with COVID-19 patients showed more symptoms of depression $(p<0.001)$ and anxiety $(p=$ $0.01)$. Furthermore, having no kids was associated with depressive symptoms $(p=0.003)$. Whereas having kids undergoing distance learning developed higher levels of depression than those whom kids were not undergoing e-learning $(p=0.01)$.

Participants who were unsure if they got infected with the virus and did not go for COVID-19 test were more vulnerable to depression $(p<0.001)$, anxiety $(p=0.04)$, and stress $(p=0.03)$. Similarly, all subscales of DASS-21 were high among HCWs who were worried about their loved ones and families during the COVID-19 crisis $(p<$ $0.001, p=0.004$ and 0.02 , respectively). Additionally, HCWs who reported that COVID-19 pandemic affected them personally were more likely to be depressed ( $p<$ $0.001)$ and stressed $(p=0.03)$. Moreover, HCWs who were compelled to change their accommodations reported higher levels of depression $(p=0.02)$, while those who were partially compelled to leave their homes were more likely to be anxious rather than depressed $(p=0.04)$. Gender had no influence on the mental health status during the COVID-19 pandemic. On the other hand, none of the sociodemographics showed an impact on the Insomnia Scale Index.

Multivariate linear regression analysis revealed that age is a major independent factor affecting all subscales of DASS-21, whereby younger adults showed significantly higher levels of depression $(p<0.001)$, anxiety $(p=$ $0.009)$, and stress $(p=0.006)$. In addition, being worried about family and loved one influenced depression $(p<$ $0.001)$ and anxiety $(p=0.016)$ independently as shown in Table 5. 
Table 3. Categorical classifications of the psychometric instruments (DASS-21 and ISI)

\begin{tabular}{|c|c|c|c|c|c|c|c|}
\hline \multicolumn{2}{|c|}{ DASS-21 depression } & \multicolumn{2}{|l|}{ DASS-21 anxiety } & \multicolumn{2}{|l|}{ DASS-21 stress } & \multicolumn{2}{|l|}{ ISI } \\
\hline category & $N(\%)$ & category & $N(\%)$ & category & $N(\%)$ & category & $N(\%)$ \\
\hline Normal & $94(23.5)$ & Normal & $85(21.3)$ & Normal & $146(36.5)$ & Not significant & $213(53.3)$ \\
\hline Mild & $41(10.3)$ & Mild & $23(5.8)$ & Mild & 69 (17.3) & Subthreshold & $134(33.5)$ \\
\hline Moderate & $63(15.8)$ & Moderate & $54(13.5)$ & Moderate & $64(16.0)$ & Moderately severe & $44(11.0)$ \\
\hline Severe & $51(12.8)$ & Severe & $27(6.8)$ & Severe & $49(12.3)$ & Severe & $9(2.3)$ \\
\hline Extremely severe & $151(37.8)$ & Extremely severe & $211(52.8)$ & Extremely severe & $72(18.0)$ & & \\
\hline
\end{tabular}

DASS-21, Depression Anxiety Stress Scale 21; ISI, Insomnia Severity Index.

Fig. 1. Depression categories among HCWs in fever, clean, and mixed clinics. HCWs, health-care workers.

Fig. 2. Anxiety categories among HCWs in fever, clean, and mixed clinics. HCWs, health-care workers.
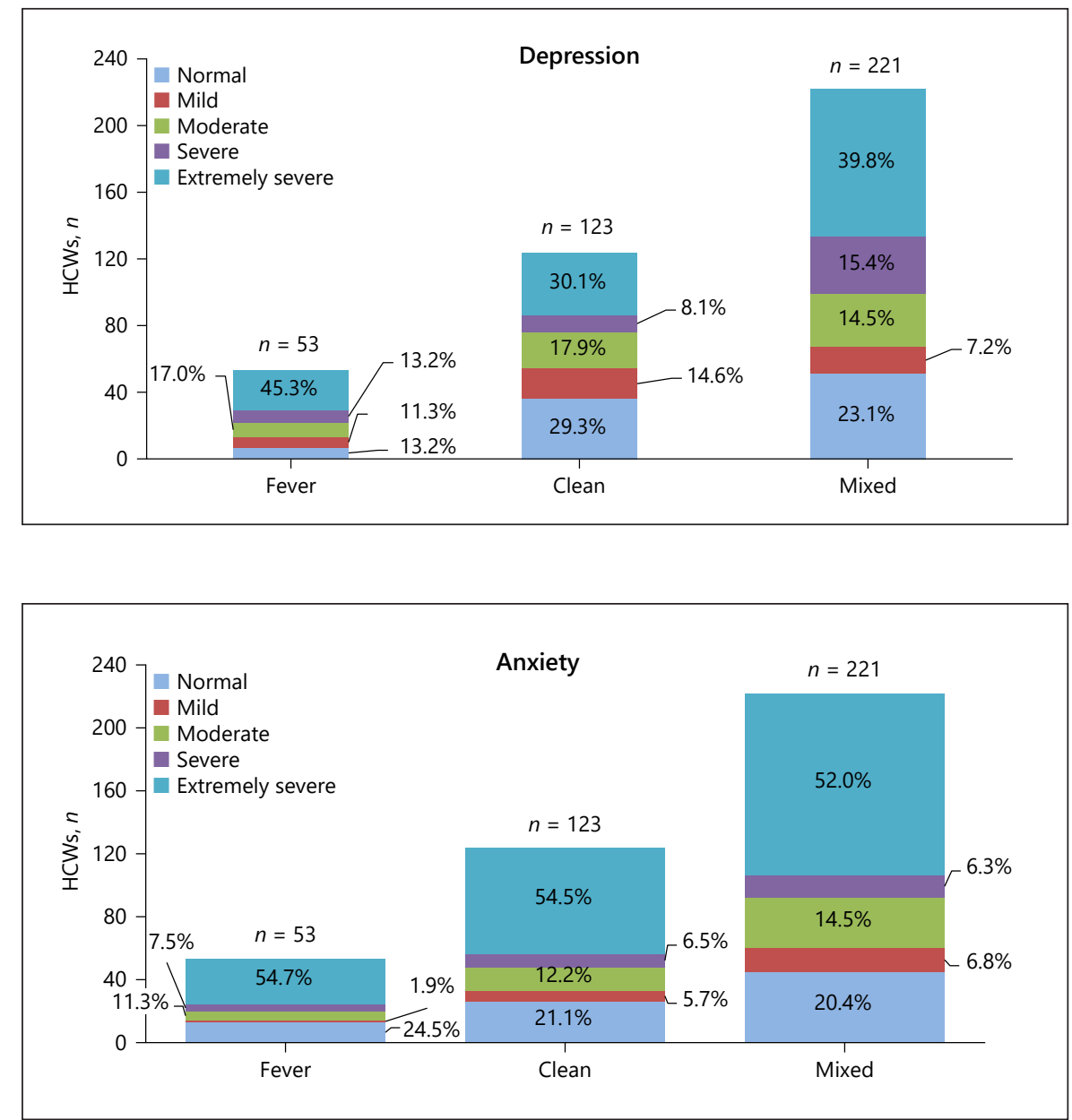

\section{Discussion/Conclusion}

The first COVID-19 cases in the UAE were confirmed in January 2020. As the virus spread and numbers increased dramatically over the world, the UAE has implemented immediate strategies to engage its health-care system in adapting and encountering this crisis. Many health-care units and inpatients beds were converted to COVID-19 related wards to incur the numerous numbers of cases in the country.

The present cross-sectional study assessed the psychometric consequences among a large number of HCWs in 4 different categories of primary health-care centers (fever, clean, telemedicine, and mixed clinics) in Dubai dur- 
Fig. 3. Stress categories among HCWs in fever, clean, and mixed clinics. HCWs, health-care workers.
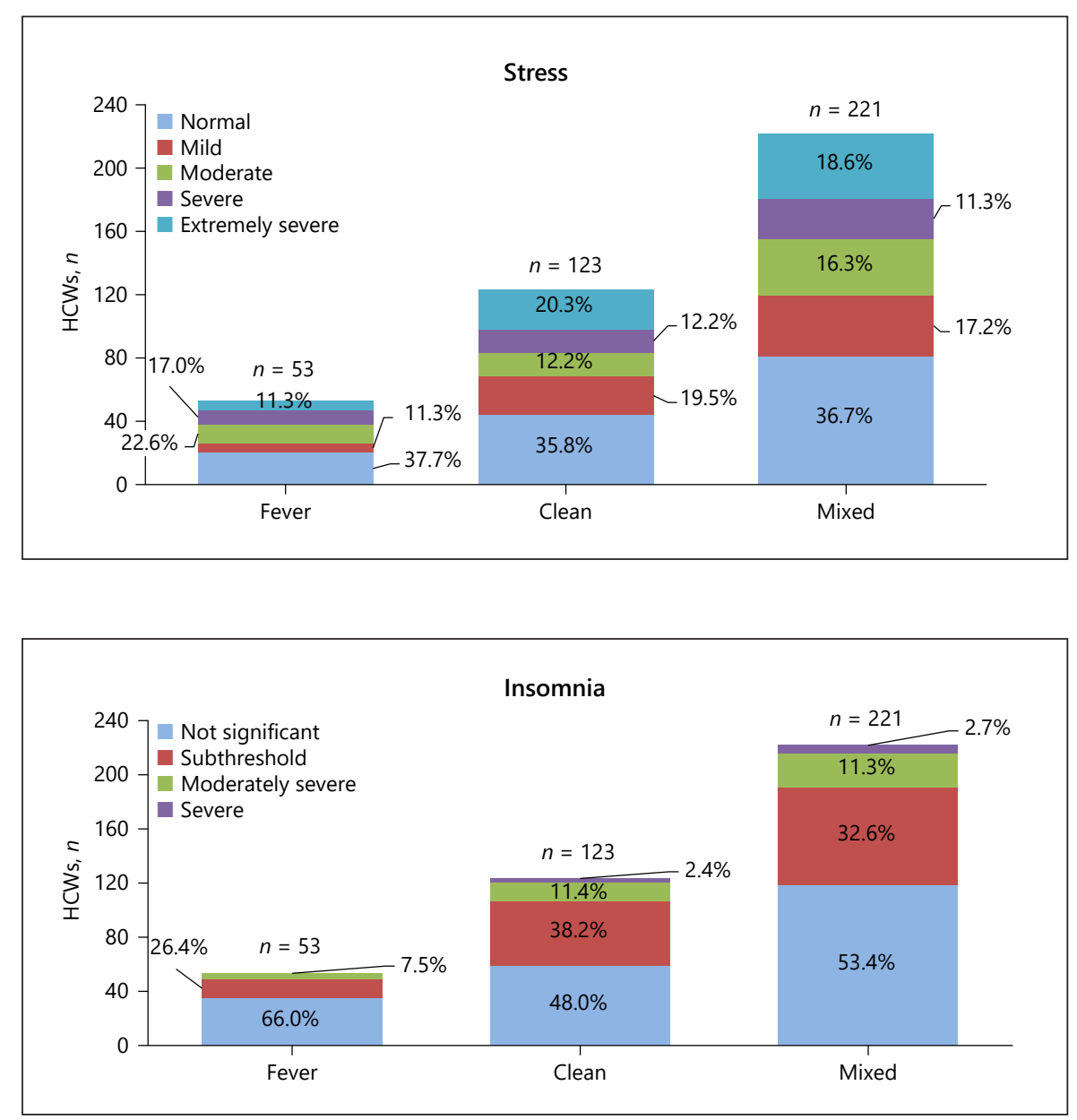

Fig. 4. Insomnia categories among HCWs in fever, clean, and mixed clinics. HCWs, health-care workers. tremely severe depressive and anxiety symptoms, but mild- to moderate-stress symptoms. Recent studies in China and Turkey had consistent results to our findings, but their participants had lower degree of severity of the mentioned psychological measures $[4,11]$. On the other hand, our study showed that a considerable proportion of HCWs during this pandemic suffered from insomnia, where only one-third $(33.5 \%, n=134)$ of them reported symptoms of subthreshold insomnia during the pandemic. This supports the finding of the China study, as insomnia was less commonly reported by the HCWs than depression and anxiety [9]. In addition, several studies during previous outbreaks reported high risk of psychological distress among the HCWs [12-14].

Our study revealed that gender has no influence on the burnout symptoms among the HCWs during the COVID-19 pandemic. In contrast, previous studies reported that women HCWs were more prone to depression and distress than men HCWs $[4,11]$. In addition, being married and having kids reduced the chance of developing 
Table 4. Factors affecting mental health of HCWs during COVID-19 crisis

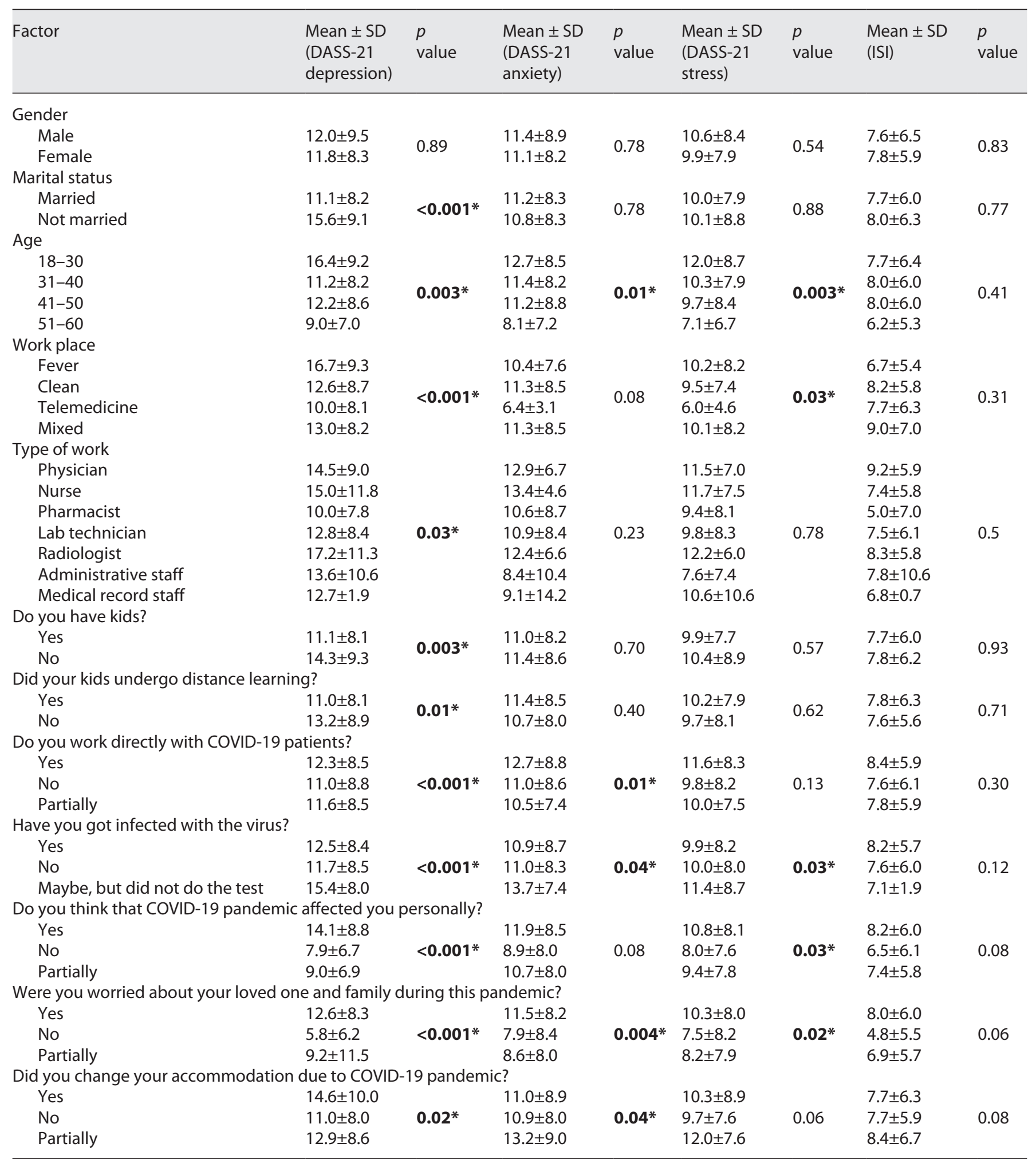

* Significance is considered at $p$ value of $\leq 0.05$. DASS-21, Depression Anxiety Stress Scale 21; ISI, Insomnia Severity Index; HCWs, healthcare workers; SD, standard deviation.

The Impact of COVID-19 on Health-Care Workers' Mental Health
Dubai Med J 2021;4:301-309

DOI: $10.1159 / 000518717$ 
Table 5. Adjusted linear regression analysis of the psychological symptoms

\begin{tabular}{|c|c|c|c|}
\hline $\begin{array}{l}\text { Multivariate Analysis } \\
\text { Independent factors }\end{array}$ & $\begin{array}{l}\text { Regression } \\
\text { coefficient }(\beta)\end{array}$ & $\begin{array}{l}\text { Significance } \\
\text { ( } p \text { value) }\end{array}$ & $\begin{array}{l}\mathrm{Cl} \\
(95.0 \%)\end{array}$ \\
\hline \multicolumn{4}{|l|}{ Depression } \\
\hline Were you worried about your loved one and family during this pandemic? & 2.73 & $<0.001$ & $1.31-4.16$ \\
\hline \multicolumn{4}{|l|}{ Anxiety } \\
\hline Age & -1.30 & 0.009 & -2.27 to -0.33 \\
\hline \multicolumn{4}{|l|}{ Stress } \\
\hline Age & -1.30 & 0.006 & -2.23 to -0.37 \\
\hline
\end{tabular}

burnout symptoms during this pandemic. This finding supported the results of a previous study, by which lower scores of DASS-21 were reported by these groups [4]. Whereas, young adults (18-30 years old) and single HCWs had higher scores of DASS-21 reflecting high levels of depression and distress among these groups. Similarly, previous studies reported that younger and single staff in the hospitals was at higher risk of having burnout symptoms during the COVID-19 and SARS outbreaks $[4,15,16]$. Furthermore, our study declared that nurses and frontline HCWs working directly with COVID-19 patients at either fever or mixed clinics were more likely to suffer from burnout symptoms. These findings are convincing as this category of people is at high risk to be exposed to the infection and have frequent contact with the patients [17, 18]. Also, this pandemic has affected the frontline HCWs personally as majority of them were worried about their families, and some were compelled to change their accommodations, which increased the risk of developing burnout symptoms. Moreover, nurses are highly responsible in conducting a high-quality nursing care for the patients and have closer contact with the patients than physicians and any other specialty in health-care systems [18-20]. Thereby, particular attention to frontline HCWs, especially the nurses treating the patients with COVID-19, should be directed regarding their psychological health and well-being. Based on the adjusted linear regression analysis of this study, being at younger age and worried about loved ones and family were the only independent factors influencing high levels of depression and anxiety.

The present study emphasis the psychological burnout followed by the COVID-19 pandemic among the HCWs, subsequently its impact on their personal and social life as well as on patients' care. Therefore, health-care orga- nizations are responsible of providing all resources to maintain safe environment for all HCWs and protecting them by implementing effective strategies and control measure for infection prevention. In addition, emotional support evidence-based training for the HCWs is required to improve their skills and experience as well as to prepare them to encounter the pandemic outbreak.

\section{Limitations}

This study has some limitations as majority of participants were women and married. Also, the specialties were not equally distributed among the HCWs; most of them were either nurses or physicians with minor numbers of other specialties. Last, it was difficult to identify those HCWs with pre-existing burnout symptoms from those who had new depression and distress during the pandemic.

\section{Conclusion}

In conclusion, this study demonstrated that nurses and physicians working at fever or mixed clinics during the COVID-19 outbreak were at high risk of developing psychological symptoms, such as depression, anxiety, stress, and insomnia. Thereby, it is important to direct efficient support and interventions to protect HCWs from being infected and promote their well-being and mental health through introducing psychological training and development programs.

\section{Acknowledgment}

The research team would like to thank the primary health-care centers (Al-Khawaneej, Al-Badda, Al-Mamzar, Nad-AlHammar, and AlMizher clinics) in Dubai Health Authority for the cooperation in conducting this study. 


\section{Statement of Ethics}

Published research must comply with the guidelines for human studies and should include evidence that the research was conducted ethically in accordance with the World Medical Association Declaration of Helsinki. The study was approved by the Dubai Scientific Research Ethics Committee in Dubai Health Authority (DSREC-06/2020_57). An online informed consent was obtained from all participants of the study. The participants have consented to the submission of the study for publication.

\section{Conflict of Interest Statement}

The authors have no conflicts of interest to declare.

\section{Author Contributions}

- F.A. (Family Medicine Physician) made substantial contributions to the conception and design of the work.

- R.A. and O.A. (Family Medicine Physicians) revised the work critically for important intellectual content and approved the version to be published.

- S.Z.A. (Clinical Pharmacist/Research Assistant) and M.M.N. (Research Assistant/Master student) drafted the work and made substantial contributions to the analysis and interpretation of data.

\section{Data Availabilty Statement}

The data being reported are accurate and are coming from the official source. Code availability: the data that support the findings of this study are available from the corresponding authors upon reasonable request.

\section{References}

1 Li Q, Guan X, Wu P, Wang X, Zhou L, Tong $\mathrm{Y}$, et al. Early transmission dynamics in $\mathrm{Wu}-$ han, China, of novel coronavirus: infected pneumonia. N Engl J Med. 2020;382:11991207.

2 WHO. Coronavirus Disease (COVID-19) situation reports [Internet]. Coronavirus Disease COVID-19 weekly epidemiological update weekly operational update. 2020 [cited 2020 Sep 26]. Available from: https://www. who.int/emergencies/diseases/novel-coronavirus-2019/situation-reports.

3 Worldometer. Coronavirus update (live): 32,806,195 cases and 994,351 deaths from COVID-19 virus pandemic: Worldometer [Internet]. COVID-19 coronavirus pandemic. 2020 [cited 2020 Sep 26]. Available from: https: //www.worldometers.info/coronavirus/.

4 Elbay RY, Kurtulmuş A, Arpacıoğlu S, Karadere E. Depression, anxiety, stress levels of physicians and associated factors in Covid-19 pandemics. Psychiatry Res. 2020;290:113130.

5 Chan AO, Huak CY. Psychological impact of the 2003 severe acute respiratory syndrome outbreak on health care workers in a medium size regional general hospital in Singapore. Occup Med. 2004;54(3):190-6.

6 DHA. DHA licensed 3,957 health care professionals and 321 health facilities in 2020 [Internet]. Dubai: Dubai Health Authority; 2020 [cited 2021 Jun 15]. Available from: https:// www.dha.gov.ae/en/DHANews/Pages/ DHANews1112608321-01-03-2021.aspx.
7 Raosoft. Sample size calculator [Internet]. [cited 2021 May 25]. Available from: http:// www.raosoft.com/samplesize.html.

8 Lovibond PF, Lovibond SH. The structure of negative emotional states: comparison of the Depression Anxiety Stress Scales (DASS) with the beck depression and anxiety inventories. Behav Res Ther. 1995 Mar 1;33(3):335-43.

9 Coker AO, Coker OO, Sanni D. Psychometric properties of the 21-item Depression Anxiety Stress Scale (DASS-21). Afr Res Rev. 2018; 12(2):135-42.

10 Bastien $\mathrm{CH}$, Vallières A, Morin CM. Validation of the Insomnia Severity Index as an outcome measure for insomnia research. Sleep Med. 2001 Jul;2(4):297-307.

11 Lai J, Ma S, Wang Y, Cai Z, Hu J, Wei N, et al. Factors associated with mental health outcomes among health care workers exposed to coronavirus disease 2019. JAMA Netw Open. 2020;3(3):e203976.

12 Lu YC, Shu BC, Chang YY, Lung FW. The mental health of hospital workers dealing with severe acute respiratory syndrome. Psychother Psychosom. 2006;75(6):370-5.

13 Lee AM, Wong JG, McAlonan GM, Cheung V, Cheung C, Sham PC, et al. Stress and psychological distress among SARS survivors 1 year after the outbreak. Can J Psychiatry. 2007;52(4):233-40.
14 Chua SE, Cheung V, Cheung C, McAlonan GM, Wong JW, Cheung EP, et al. Psychological effects of the SARS outbreak in Hong Kong on high-risk health care workers. Can J Psychiatry. 2004;49(6):391-3.

15 Liu X, Kakade M, Fuller CJ, Fan B, Fang Y, Kong J, et al. Depression after exposure to stressful events: lessons learned from the severe acute respiratory syndrome epidemic. Compr Psychiatry. 2012;53(1):15-23.

16 Wu P, Fang Y, Guan Z, Fan B, Kong J, Yao Z, et al. The psychological impact of the SARS epidemic on hospital employees in China: exposure, risk perception, and altruistic acceptance of risk. Can J Psychiatry. 2009;54(5): 302-11.

17 Li L, Cheng S, Gu J. SARS infection among health care workers in Beijing, China. JAMA. 2003;290(20):2662-3.

18 Shih F-J, Gau M-L, Kao C-C, Yang C-Y, Lin $\mathrm{Y}-\mathrm{S}$, Liao Y-C, et al. Dying and caring on the edge: Taiwan's surviving nurses' reflections on taking care of patients with severe acute respiratory syndrome. Appl Nurs Res. 2007; 20(4):171-80.

19 Mok E, Chung BP, Chung JW, Wong TK. An exploratory study of nurses suffering from severe acute respiratory syndrome (SARS). Int $J$ Nurs Pract. 2005;11(4):150-60.

20 Tzeng H-M. Fighting the SARS epidemic in Taiwan: a nursing perspective. JONA J Nurs Adm. 2003;33(11):565-7. 